Autorität des Staatspräsidenten Dmitrij Medwedew auf derjenigen Putins gründete - seinem vom Nimbus eines durchsetzungsfähigen „starken Mannes“ umgebenen Amtsvorgänger und Vorsitzenden der Staatspartei „Geeintes Russland“.

$\mathrm{Zu}$ den semi-präsidentiellen Staatsoberhäuptern zählen auch derjenige Portugals (obwohl die Kompetenzen des Präsidenten bei der Bildung und Entlassung der Regierung 1982 stark beschnitten worden sind) und der Ukraine - beide werden kurz behandelt. Am Beispiel Finnlands und, ebenfalls in einer Kurzfassung, Kroatiens, wird dargelegt wie semipräsidentielle Systeme sich zu parlamentarischen hin entwickelt haben, am Beispiel Polens, dass die parlamentarische Komponente durch eine zurückhaltende Wahrnehmung präsidialer Befugnisse zunehmend an Gewicht gewonnen hat.

Wie die Länderstudien zeigen, ist der Amtsumfang der Staatsoberhäupter von den Verfassungen vorgegeben, wird die Amtsausübung auch vom Grad der Verankerung im Parteiensystem und vom gesellschaftlichem Umfeld beeinflusst, wird das Amtsverständnis vom jeweiligen Amtsinhaber geprägt, der seine Kompetenzen voll ausschöpfen oder aber sich auf die Wahrnehmung einer rein repräsentativen und staatsnotariellen Rolle beschränken kann. Sind Monarch und Präsident nur Staatsoberhäupter, so kommt ihnen die wichtige Aufgabe zu, für den gesellschaftlichen Konsens zu sorgen, außerhalb der politischen Agenda stehende Themen anzusprechen, den Parteien „die Leviten zu lesen“ (S. 310).

Hartmann und Kempf haben sich darum verdient gemacht, Kenntnisse über ein Staatsorgan zu vertiefen, das in Darstellungen politischer Systeme mitunter stiefmütterlich behandelt wird.

Robert K. Furtak

\title{
Parteien als Organisationen: wichtige Beiträge zur Debatte
}

Jun, Uwe und Benjamin Höhne (Hrsg.): Parteien als fragmentierte Organisationen. Erfolgsbedingungen und Veränderungsprozesse, Verlag Barbara Budrich, Opladen 2010, 282 Seiten, $€ 33,-$.

Die Parteienforschung kennt zwei Figuren, um die Organisationswirklichkeit von Parteien zu beschreiben. Einerseits werden sie als zentralisierte Organisationen charakterisiert, die mit Robert Michels dem „ehernen Gesetz der Oligarchie“ unterliegen. Andererseits - und diese Position findet sich vermehrt in der jüngeren Literatur - gelten Parteien als fragmentiert, dezentral organisiert, unbestimmt, pluralistisch oder gar als „lose verkoppelte Anarchie“ beziehungsweise „Stratarchie“ (S. 13). Wertvolle Hinweise darauf, inwieweit diese Zuschreibungen tatsächlich zutreffen und wie unter diesen Bedingungen noch strategische Steuerung und geplanter Wandel möglich sind, liefert dieser Sammelband, der die Beiträge einer Tagung des Arbeitskreises Parteienforschung der DVPW zusammenführt.

Uwe Jun (S. 11) arbeitet in einem einführenden Überblicksartikel zwei Prinzipien heraus, die innerhalb einer Partei aufeinandertreffen: die Profi- und die Freiwilligenpartei (S. 15). Während erstere an Steuerung, Professionalisierung und Wahlerfolgen orientiert ist, zeichnet sich letztere durch Partizipation, Mitgestaltung und Binnenorientierung aus (S. 16 ff.). In diversen Fallstudien und Detailanalysen zur Organisationswirklichkeit von 
Parteien zeigt der Sammelband, wo sich beide Prinzipien gegenüberstehen und wo sie Hand in Hand gehen. So berichtet Patrick Donges Ergebnisse eines größeren Forschungsprojekts (S. 71) zur Parteikommunikation. Danach stellt Kommunikation das einende Band zwischen den Parteifragmenten dar (S. 66), jedoch können die Parteien die Kommunikationsbedingungen nicht so gestalten, dass sie zur strategischen Kommunikation fähig wären (S. 70). Die Befunde, die auf einem Vergleich von insgesamt zehn Parteien in vier Ländern basieren, sind ambivalent: Anhand parteiorganisatorischer Änderungen lässt sich ein Bedeutungszuwachs der Medienarbeit sowie der Kommunikation generell erkennen (S. 80), der sich aufgrund der fragmentierten Parteistrukturen aber nicht in einer geschlossenen strategischen Kommunikation niederschlägt - wobei diese Einsichten auch ohne die vorangestellten, grundlegenden sozialtheoretischen Erörterungen zu Akteur, Struktur und Handlungssystem (S. 67 ff.) überzeugt hätten. Benjamin Höhne setzt sich mit der Frage auseinander, ob bei der Aufstellung von Kandidaten für Wahlen neue gesellschaftliche Anforderungen an Partizipation und Repräsentation Berücksichtigung erfahren (S. 90). In einer explorativen Studie zur Entwicklung der Kandidatenauswahl für das Europäische Parlament (wo die Parteispitze stärkeren Einfluss ausübt) und den Bundestag (die weitgehend in der Hand der Parteibasis liegt) findet Höhne anhand der Verteilung sozialstruktureller Merkmale einige Bestätigung für die These, dass Parteieliten bei der Auswahl von Kandidaten stärker auf deren elektoralen Erfolgsaussichten achten (S. 92 f.). Allerdings legt der Autor zugleich die Grenzen seiner Studie offen: Es wird nur der Einfluss der Nominierungsbedingungen betrachtet, notwendig wäre daneben die Einbeziehung von Ressourcen und Motiven der Kandidaten (S. 115). Diese Aspekte berücksichtigt Tim Spier in einer ReAnalyse der Potsdamer Parteimitgliederstudie zur Selektion lokaler Vorstandsmitglieder. Dabei gelangt er zu dem bemerkenswerten Befund, dass die Ressourcenausstattung der Angehörigen nicht auf Orts-, sehr wohl aber auf Kreisebene eine Rolle spielt (S. 143 f.). Damit ergänzt Spier den bisherigen Stand der Forschung zur innerparteilichen Partizipation: Parteimitglieder haben - unabhängig von ihrer individuellen Ressourcenausstattung ähnliche Chancen, am Parteileben teilzunehmen; für den politischen Aufstieg sind aber die ressourcenstarken prädestiniert.

In seiner Analyse afrikanischer Parteien zeigt Alexander Stroh die Möglichkeiten und Grenzen der Übertragung von Theorien, Ansätzen und Methoden, die aus der Auseinandersetzung mit europäischen beziehungsweise westlichen Parteien entstanden sind. Im Fokus stehen die innerparteilichen Machtdynamiken in insgesamt 16 Parteien in Benin und Burkina Faso (S. 156). Mit einem explorativen Design, das Beobachtungen, Interviews und eine Indexbildung kombiniert, werden innerparteiliche Partizipation, Faktion und Loyalität untersucht. Zwei wesentliche Ergebnisse treten zutage: Erstens kann der Autor keinen Zusammenhang zwischen der internen Machtdynamik einer Partei und deren Wahlerfolg feststellen. Zweitens zeigen sich entgegen landläufiger Auffassung wesentliche Differenzen in der innerparteilichen Dynamik und im Institutionalisierungsgrad der untersuchten Parteien. Der Autor ist ausdrücklich dafür zu loben, wie er ungeachtet der zugestandenen Datenprobleme (S. 155, S. 169) ein ansprechendes Untersuchungsdesign und eine überzeugende Analyse entwickelt. Der (vorläufige) Befund, inwieweit innerparteiliche Machtdynamiken sich auf Wahlerfolge niederschlagen, verdient auch in westlichen Parteiensystemen intensive Aufmerksamkeit.

In seinem Beitrag zu den deutschen Parteien schlägt Andreas Feser vor, deren Rechenschaftsberichte stärker als Datenbasis zu nutzen. Er vergleicht Union und SPD seit den 
1990er Jahren und kommt zu dem Schluss, dass in der SPD eine Zentralisierung der Finanzkraft stattgefunden hat. Dies ziehe, so seine diskussionswürdige These, Defizite der Kampagnenfähigkeit in den Landesverbänden nach sich, wie die Landtagswahlergebnisse der letzten Jahre belegten (S. 202). Den Folgen der Organisationsreformen von SPD und CDU auf Landesebene widmen sich Wolfgang Schroeder und Arijana Neumann. Dazu werden die unterschiedlichen Konzepte der Großparteien vorgestellt (SPD als Netzwerkpartei, CDU als Bürgerpartei) und die Konsequenzen in vier Bundesländern beobachtet. Die angedachten und festzustellenden Änderungen stehen dabei stets im Spannungsfeld von zentralisierter Professionalisierung und partizipatorischer Erneuerung und Erweiterung (S. 211). Die Analyse zeigt, dass die CDU im Vergleich stärker auf partizipative Elemente setzt (S. 223 f.). Für beide Parteien gilt aber, dass die Debatten über die Reformen weiter fortgeschritten sind als die tatsächlich eingeleiteten Maßnahmen (S. 224). Eser Kiziloglu Sönmez und Lothar Probst inspizieren in einem weiteren Beitrag, was die hessischen Landesverbände zur Professionalisierung (vorrangig verstanden als Qualifizierung) ihrer Mitglieder unternehmen (S. 229). Im Vergleich findet sich die stärkste Institutionalisierung von Fort- und Weiterbildungsmaßnahmen in der CDU und die geringste in der FDP (S. 251). Die Grenzen dieser Maßnahmen bestehen wiederum im Freiwilligencharakter der Parteien und der Unvorhersehbarkeit des politischen Betriebs. Der abschließende Beitrag von Sebastian Buckow legt den Fokus auf die Angehörigen der Parteizentralen, denen vielfach eine (neue) Bedeutung zugeschrieben wird und berichtet Ergebnisse einer innovativen Befragung (S. 267). Demnach weisen die hauptamtlichen Mitarbeiter der Parteizentralen die Ressourcen und Intentionen zur innerparteilichen Einflussnahme auf und sollten deshalb als Teil der Parteiführung künftig verstärkt ins Blickfeld der Parteiorganisationsforschung rücken (S. 273).

In seiner Beleuchtung unterschiedlicher Aspekte der Parteiorganisation vereint der Sammelband eine Reihe interessanter und diskussionswürdiger Einzelbefunde. Wie für die Parteien eine Fragmentierung diagnostiziert wird, gilt dies in Teilen auch für den Band selbst. Der Beitrag von Elmar Wiesendahl zeigt Möglichkeiten auf, wie die Einzelbefunde zusammenzubringen sind. Er diskutiert den Parteienwandel vor dem aktuellen Stand der Organisations- und Wandlungstheorien (S. 35). Seine zentrales Anliegen ist, dass die Parteienforschung stärker deren Erkenntnisse rezipiert, um Änderungen in Parteien einzuordnen, zu erklären und zu bewerten. Hierzu verbindet Wiesendahl organisations- und wandlungstheoretische Ansätze und wendet sie auf politische Parteien an. Im Ergebnis kristallisiert sich heraus, dass Parteienwandel nicht allein als Anpassung an veränderte Umweltbedingungen zu begreifen ist. Stets sind die Entscheidungen und Neuausrichtungen der Parteiführungen (und der dahinter stehende Auswahlprozess) zu betrachten (S. 56). Damit gerät zunehmend die Interaktion der innerparteilichen Akteursebene - und zwar von Parteieliten und Mitgliedern gleichermaßen - in den Fokus des Interesses, um zu einer angemessenen Charakterisierung der Organisationswirklichkeit und des Wandels von Parteien zu gelangen. 